\title{
Genomic Profiling of Chronic Myelogenous Leukemia: Basic and Clinical Approach
}

REVIEW

\author{
Aliasghar Keramatinia ${ }^{1}$, Alireza Ahadi ${ }^{1,2}$, Mohammad Esmaeil Akbari ${ }^{1}$, Maryam Mohseny ${ }^{3}$, Alireza Mosavi Jarahi ${ }^{3}$, \\ Narjes Mehrvar ${ }^{1}$, Neda Mansouri ${ }^{1,2}$, S.A. Mortazavi Tabatabaei ${ }^{4}$, Abolfazl Movafagh ${ }^{1,2}$ \\ ${ }^{1}$ Cancer Research Center, Shahid Beheshti University of Medical Sciences, Departments of ${ }^{2}$ Medical Genetics and ${ }^{3}$ Social Medicine, School of \\ Medicine, Shahid Beheshti University of Medical Sciences, ${ }^{4}$ Proteomics Research Center, School of Paramedical Sciences, Shahid Beheshti University \\ of Medical Sciences, Tehran, Iran
}

\begin{abstract}
Chronic myeloid leukemia (CML) is a hematological stem cell cancer driven by BCR-ABL1 fusion protein. We review the previous and recent evidence on the significance of $C M L$ in diagnostic and clinic management. The technical monitoring of $B C R-A B L 1$ with quantitative real time-PCR has been used in assessing patient outcome. The cytogenetic mark of CML is Philadelphia chromosome, that is formed by reciprocal chromosomal translocations between human chromosome 9 and $22, t(9: 22)\left(q^{34}: q^{11}\right)$. It makes a BCR-ABL1 fusion protein with an anomaly tyrosine kinase activity that promotes the characteristic proliferation of progenitor cells in CML and acute lymphoblastic lymphoma. The targeting of BCR-ABL1 fusion kinase is the first novel paradigm of molecularly targeted curing
\end{abstract}

(J Cancer Prev 2017;22:74-81)

Key Words: Chronic myeloid leukemia, Profile, Methods, BCR-ABL1

\section{INTRODUCTION}

Chronic myeloid leukemia (CML) has been considered as one of the essential neoplastic aberration directly linked to a genetic disorder. CML is a three-phase disease. Most of the patients diagnosed with CML are in a chronic phase (CP) with $<10 \%$ myeloblasts in the bone marrow (BM) and blood. Untreated CML-CP invariably transforms into blastic phase (BP) resembling acute myeloid and lymphoid leukemia with $>20 \%$ myeloblasts in the bone marrow and peripheral blood. Intermediate phase, named accelerated phase, is defined with $10 \%$ to $20 \%$ myeloblasts. ${ }^{1}$ The karyotype mark of $\mathrm{CML}$ is Philadelphia $\left(\mathrm{Ph}^{1}\right)$ chromosome, ${ }^{2}$ which is a result of chromosomal rearrangement, $\mathrm{t}(9: 22)\left(\mathrm{q}^{34}: \mathrm{q}^{11}\right) .^{3}$ Aberrant BCR-ABL1 fusion protein constitutively activates downstream enzymes which enhance growth factor-independent proliferation, reprogramed adhesion, and resistance to DNA repair. The transcription of $B C R-A B L$ and the resulting fusion protein is a key target and biomarker in the treatment and monitoring of CML (Fig. 1). The targeting of BCR-ABL fusion kinase is the prime novel paradigm of molecularly targeted therapy. Tyrosine kinase inhibitors (TKIs) that specifically inhibit BCR-ABL signaling activity by competing for ATP may revolutionize the treatment of $\mathrm{CML}^{4}$

The tyrosine kinase activated by fusion changes the levels of enzyme phosphorylation and inhibits cellular apoptosis, which are essential for initiating malignancy. TKI effectively inhibits the activity of the BCR-ABL1 proteins in patients with CML by attaching to the ATP-binding pocket of tyrosine kinase ${ }^{5}$ and managing minimal residual disease and target relapse after curing. ${ }^{6}$ Responses to TKI therapy are determined with changes in

Received May 17, 2017, Revised June 10, 2017, Accepted June 12, 2017

Correspondence to: Abolfazl Movafagh

Department of Medical Genetics, School of Medicine, Cancer Research Center, Shohada Referral Hospital, Shahid Beheshti University of Medical Sciences, Tehran, Iran

Tel: +98-21-2240067, E-mail: movafagh.a@sbmu.ac.ir

*This research study is part of Aliasghar Keramatinia PhD thesis work.

Copyright (C) 2017 Korean Society of Cancer Prevention

(c) This is an Open Access article distributed under the terms of the Creative Commons Attribution Non-Commercial License (http://creativecommons.org/licenses/by-nc/4.0) which permits unrestricted non-commercial use, distribution, and reproduction in any medium, provided the original work is properly cited. 
A

Ph chromosome

q34 \{

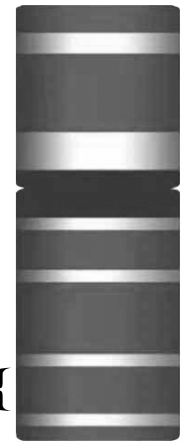

9

$A B L 1$

q11 \{

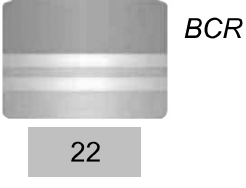

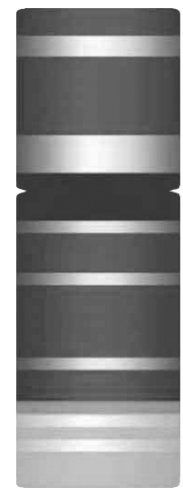

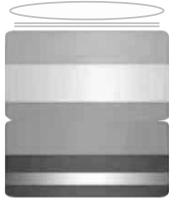

$B C R$

$A B L 1$

$A B L 1$

$B C R$

\section{$9 q^{+}$}

B

$B C R-A B L 1$ fusion transcripts

$A B L 1 \quad \operatorname{la}_{\mathrm{a}}^{\mathrm{b}} \quad \mathrm{a} 2 \mathrm{a}$

a11

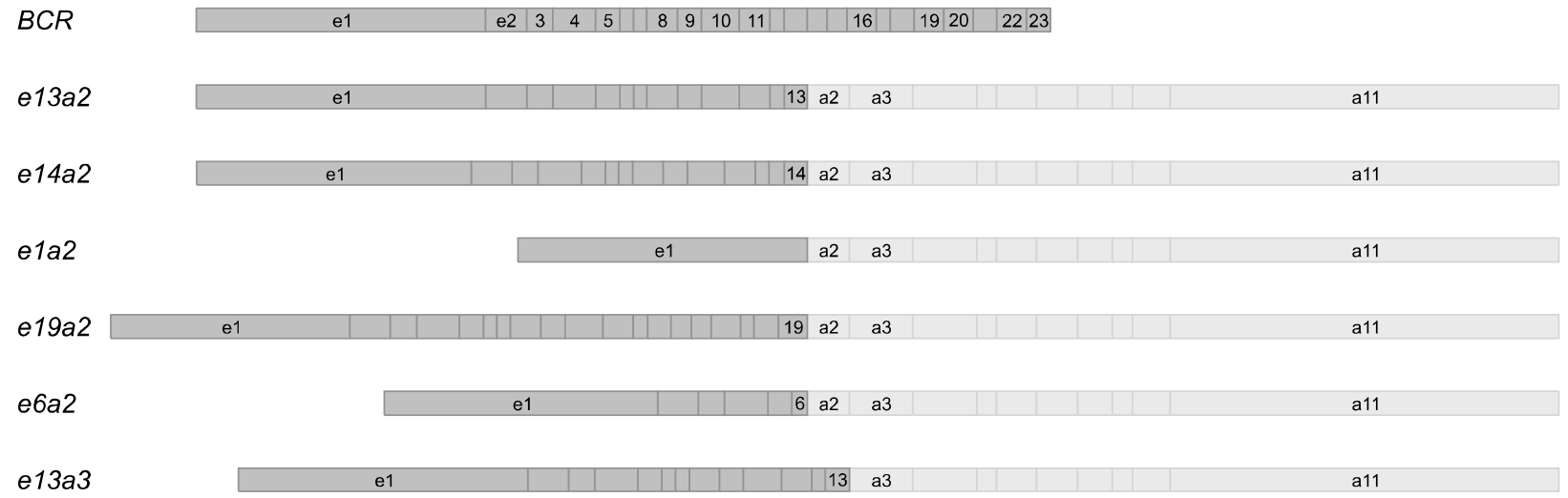

Figure 1. Schematic view of Philadelphia $\left(\mathrm{Ph}^{1}\right)$ chromosome and $B C R-A B L 1$ fusion transcripts. (A) The $\mathrm{Ph}^{1}$ chromosome is the result of $\mathrm{t}(9 ; 22)\left(\mathrm{q}^{34}: \mathrm{q}^{11}\right)$. (B) $B C R-A B L 1$ fusion transcripts with different breakpoints (https://en.wikipedia.org/wiki/File:Schematic_of_the_Philadelphia_ Chromosome.svg).

BCR-ABL fusion protein.

\section{ELEMENTS TO DETECT BCR-ABL1 GENE AND PROTEIN}

The detection of $B C R-A B L 1$ and its product has been achieved through a series of development from karyotyping to cellular and molecular genetics. However, its sensitivity is not sufficient to diagnose total chromosomal aberration. In contrast, immunologic methods are useful to diagnose the onco-protein in peripheral blood specimen based on antibody-antigen binding. The labeled antibodies bind to fusion proteins and the signals are detected by colorimetric and flow cytometric analyses to measure fusion amino acid. ${ }^{7}$

\section{CYTOGENETIC ANALYSIS}

Cytogenetic method shows metaphase stage by banding methods that subdivide each interphase into many of alternating white and black bands. ${ }^{8}$ The bone marrow specimen from individual was cultured to obtain metaphase in the slide. After specific staining procedure (e.g., Giemsa, quinacrine mustard), the arrangement of metaphases can be visualized with a fluorescence microscope.

Fluorescence in situ hybridization (FISH) can detect cells in metaphase or interphase, and specimens can derive from bone marrow, peripheral blood, and other specimen. These probes are fluorochrome-labeled and the detected signals are observed with a fluorescence microscope. ${ }^{9}$ Normally, there is red $B C R$ signal (R) and green $A B L$ signal (G) on the normal chromosomes 9 and 22 , 
respectively. Compared with conventional cytogenetics, FISH needs less time to get results (often within 45 hours) and has high sensitivity to detect specific disorder.

\section{NATIONAL COMPREHENSIVE CANCER NETWORK GUIDELINES ON MONITORING FREQUENCIES}

The National Comprehensive Cancer Network (NCCN) (Table 1) has provided and updated guidelines for the frequencies of molecular monitoring in patients treated with TKIs.

\section{Molecular methods}

PCR is used widely to measure $B C R-A B L 1 \mathrm{mRNA}$ for its high sensitivity and specificity. Total RNA is extracted from blood or $\mathrm{BM}$ and then reverse transcribed into CDNA using specific primers through PCR reaction. ${ }^{10}$ If the first PCR reaction has nonspecific products, the products cannot be amplified in the second reaction. The specificity of $B C R-A B L 1$ diagnostic assays could be enhanced by nested primers. PCR products are observed by agarose gel electrophoresis to identify whether $B C R-A B L$ transcripts are detectable.

\section{Somatic mutations in epigenetic regulators}

Some mutations in the genes of epigenetic regulators, ASXL1, TET2, TET3, KDM1A, and MSH6 were found in 25\% of patients. DNA methylation has reported to be associated with pathogenesis of CML. ${ }^{9}$ Three loss-of-function mutations (frameshift insertion, deletion, or nonsense mutation) found in ASXL1 were existed within exon $12 .^{10}$
ASXL1 has well-known functions in histone modification and plays a role as a putative tumor suppressor that is often reported to be mutated in hematological malignancies. In myelodysplastic syndrome/myeloproliferative neoplasm (MDS/MPN) patients, ASXL1 mutations were concentrated within exon 12. ${ }^{11,12}$ Frameshift or nonsense mutations in exon 12 of $A S X L 1$ lead to the truncation of the protein and removal of the C-terminal, which contains a PHD finger that is a structural motif found in nuclear proteins and is involved in transcriptional regulation, chromatin modifications, and histone demethylation. ${ }^{12-15}$

TET2 initiates DNA demethylation and is frequently mutated in hematological malignancies, including CML. ${ }^{16}$ TET2 mutations in JAK2 mutation-positive MPN patients clarify the effect of mutation order on disease phenotype and progression. KDM1A (also known as lysine-specific demethylase 1 ) is associated with the maintenance and differentiation of hematopoietic stem cells by demethylation of di-and tri-methylation of lysine 4 on histone 3. ${ }^{17}$

MSH6 is an essential component of the DNA mismatch repair mechanism. ${ }^{18}$ Loss-of-function mutation was reported in relapsed ALL patients and the mutation leads to constitutional mismatch repair deficiency syndrome, which is characterized by the development of childhood cancers (Table 2), mainly hematological malignancies. ${ }^{19}$ Taken together, epigenetic regulation may play important roles against the etiology of CML.

Recurrent somatic mutations in COL7A1, CSMD2, CLSTN2, and $D Y S F$ were also found in two patients. It has been reported that COL7A1 expression was significantly upregulated in cancer stem cells of solid tumors by the positive stimulation of TGF- $\beta 1$ signaling. ${ }^{20}$ TGF- $\beta$ is a critical regulator of Akt activation in

Table 1. NCCN guidelines on chronic myelogenous leukemia

\begin{tabular}{lll}
\hline \multicolumn{1}{c}{ Indication } & \multicolumn{1}{c}{ Testing } & \multicolumn{1}{c}{ Frequency } \\
\hline At diagnosis before therapy & RT-qPCR and BM cytogenetics & \\
& If BM is not feasible, FISH on PB is acceptable & \\
During therapy & RT-qPCR & Every 3 months \\
& BM cytogenetics & BM cytogenetics At 3, 12, and 18 months \\
After complete cytogenetic response & ABL kinase domain mutation analysis & When initial response is inadequate \\
& RT-qPCR & Every 3-6 months \\
& BM cytogenetics to detect clonal evolution & As clinically indicated \\
& FISH is not recommended & As clinically indicated \\
Increasing levels of $B C R-A B L 1$ transcripts & Evaluate compliance & \\
& Repeat RT-qPCR in those with MMR & \\
& BM cytogenetics in those without MMR & \\
& Consider ABL kinase domain mutation analysis & \\
\hline
\end{tabular}

National Comprehensive Cancer Network (NCCN) Clinical Practice Guidelines in Oncology (NCCN Guidelines) Chronic Myelogenous Leukemia version 3.2013 (section CML-A, page 15; http://www.nccn.org/professionals/physician_gls/pdf/cml.pdf). RT-qPCR, quantitative real time-PCR; BM, bone marrow; $\mathrm{PB}$, peripheral blood; MMR, mismatch repair. 
Table 2. Relative frequencies of recently identified molecular abnormalities in chronic myelomonocytic leukemia

\begin{tabular}{cclc}
\hline $\begin{array}{c}\text { Major class of } \\
\text { genetic mutation }\end{array}$ & Classify & Gene & $\begin{array}{c}\text { Frequency of } \\
\text { mutation (\%) }\end{array}$ \\
\hline Epigenetic control & $\begin{array}{c}\text { Histone } \\
\text { modification }\end{array}$ & ASXL1 & 40 \\
& DNA & EZH2 & $5-10$ \\
& methylation & TET2 & $40-60$ \\
& & DNMT3A & $<5$ \\
& BOTH & IDH1 & 1 \\
& & IDH2 & $5-10$ \\
Cytokine signaling & & JAK2 & $5-10$ \\
& & NRAS & 10 \\
& & KRAS & $5-10$ \\
& & NOTCH2 & $<5$ \\
Pre-mRNA splicing & & FLT3 & $<5$ \\
& & SRSF2 & $30-50$ \\
Transcription and & SF3B1 & $5-10$ \\
nucleosome assembly & & U2AF1 & $<5$ \\
DNA damage & RUNX1 & 15 \\
& & & \\
& & SETBP1 & $5-10$ \\
& & TP53 & 1 \\
\hline
\end{tabular}

leukemia-initiating cells and controls FOXO3A localization in CML, which is responsible for maintaining leukemia-initiating cells.

\section{Standardization of the multistep quantitative real time-PCR}

For molecular testing results to become practical information that affects management decisions, it is imperative to standardize quantitative real time PCR (RT-qPCR) to permit comparison of individual laboratory results to the IRIS data and to enable clinical practice to follow NCCN guidelines. ${ }^{5}$ RT-qPCR is a technically challenging multistep technique (Fig. 2). Differences in sample collection, cell preparation, RNA isolation, reverse transcription, internal control selection, standard curve construction, and data reporting contribute to the outstanding variation found in the reported $B C R-A B L 1$ data.

\section{Histopathology and immunohistochemistry}

There is predominance of the granulocytic lineage, with dysgranulopoiesis as a defining feature of the disease. However, the presence of eosinophilia in the absence of $B C R-A B L 1$ transcripts should alert the pathologist for a possible rearrangement of PDGFRA, PDGFRB, or fibroblast growth factor receptor-1 overexpression. Bone marrow fibrosis may be present in up to $30 \%$ of patients with chronic myelomonocytic leukemia

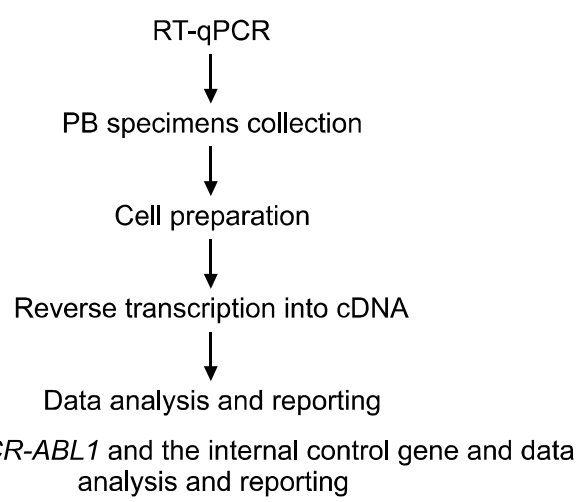

Figure 2. The principal steps of the quantitative real time-PCR (RT-qPCR) procedure. PB, peripheral blood.

(CMML). The peripheral blood and bone marrow monocytes usually express $\mathrm{CD} 33$ and $\mathrm{CD} 13$, the typical antigens on myelocytes.

\section{CHROMOSOMAL AND MOLECULAR ABNORMALITIES}

No specific cytogenetic alterations have been identified in chromosome banding analysis of patients with CML. There has been an explosion in the discovery of several novel molecular abnormalities in patients with CMML. These can be divided into the following categories: (a) mutations in epigenetic control of transcription, ${ }^{15,21-25}$ such as histone modification (EZH2, ASXL1, and UTX), DNA methylation (TET2 and DNMT3A), or both (IDH1 and IDH2), (b) mutations in the spliceosome machinery (SF3B1, SRSF2, U2AF35 and U2AF65), (c) mutations in genes that regulate cytokine signaling (JAK2, KRAS, NRAS, CBL, and FLT3), ${ }^{26-28}$ (d) mutations in transcription factors and nucleosome assembly (RUNX1 and SETBP1), ${ }^{29-31}$ and (e) mutations in DNA damage response genes, such as $T P 53^{18}$ The relative frequency of these mutations in individuals with CMML is shown in Table 2. The most commonly identified abnormalities (approximately 40\%-60\% of all CMML patients) are mutations in ASXL1, SRSF2, and TET2genes. Mutations in NRAS, CBL, IDH2, RUNX1, SETBP1, and SRSF2 have been shown to be predictors of poor outcome in univariable analysis. In contrast, mutations in the $A S X L 1$ gene have been shown to be significant in multivariable analysis. ${ }^{32}$

\section{HEDGEHOG SIGNALING AND CANCER}

The transition from the chronic to the advanced phase of CML involves distinct changes in gene expression that predict increased activation of the WNT/ $\beta$-catenin pathway, as well as 
deregulated expression of several transcriptional regulators, including JunB, Fos, and PRAME. ${ }^{18}$ Imatinib treatment has clearly improved the prognosis for CML patients, especially in the $\mathrm{CP}$, the occurrence of relapse, ${ }^{1,2}$ resistance, ${ }^{23,33,34}$ and the requirement for continued therapy. ${ }^{35,36}$ Here, we examine the stem cell origin of $\mathrm{CML}$, and then discuss the recent findings that the Hedgehog $(\mathrm{Hh})$ pathway contributes to the survival and expansion of $B C R-A B L$ leukemic stem cells. ${ }^{37,38}$ Further analysis of patients resistant to imatinib led to the discovery of $B C R$ - $A B L$ amplifications and point mutations primarily located within the BCR-ABL kinase domain.

The Hh pathway, first discovered in the model organism Drosophila, functions in tissue patterning during embryonic development. In the absence of Hh ligands, the Patched (PTCH) receptor functions as an antagonist of the pathway by inhibiting the activation of Smoothened (SMO) (Fig. 3). However, when Hh ligands bind $\mathrm{PTCH}$, this effectively relieves repression on SMO, resulting in its activation. SMO activation culminates in a signal transduction cascade that causes the nuclear translocation of the GLI family of transcription factors (GLI1, 2, 3) and the subsequent induction of a distinct transcriptional regulatory program.

The role of the Hh pathway in post-natal hematopoeisis has been investigated using several experimental approaches. The expression of the two classical Hh target genes, GLI1 and PTCH1, was assayed in CML patients, as in the murine BCR-ABL bone
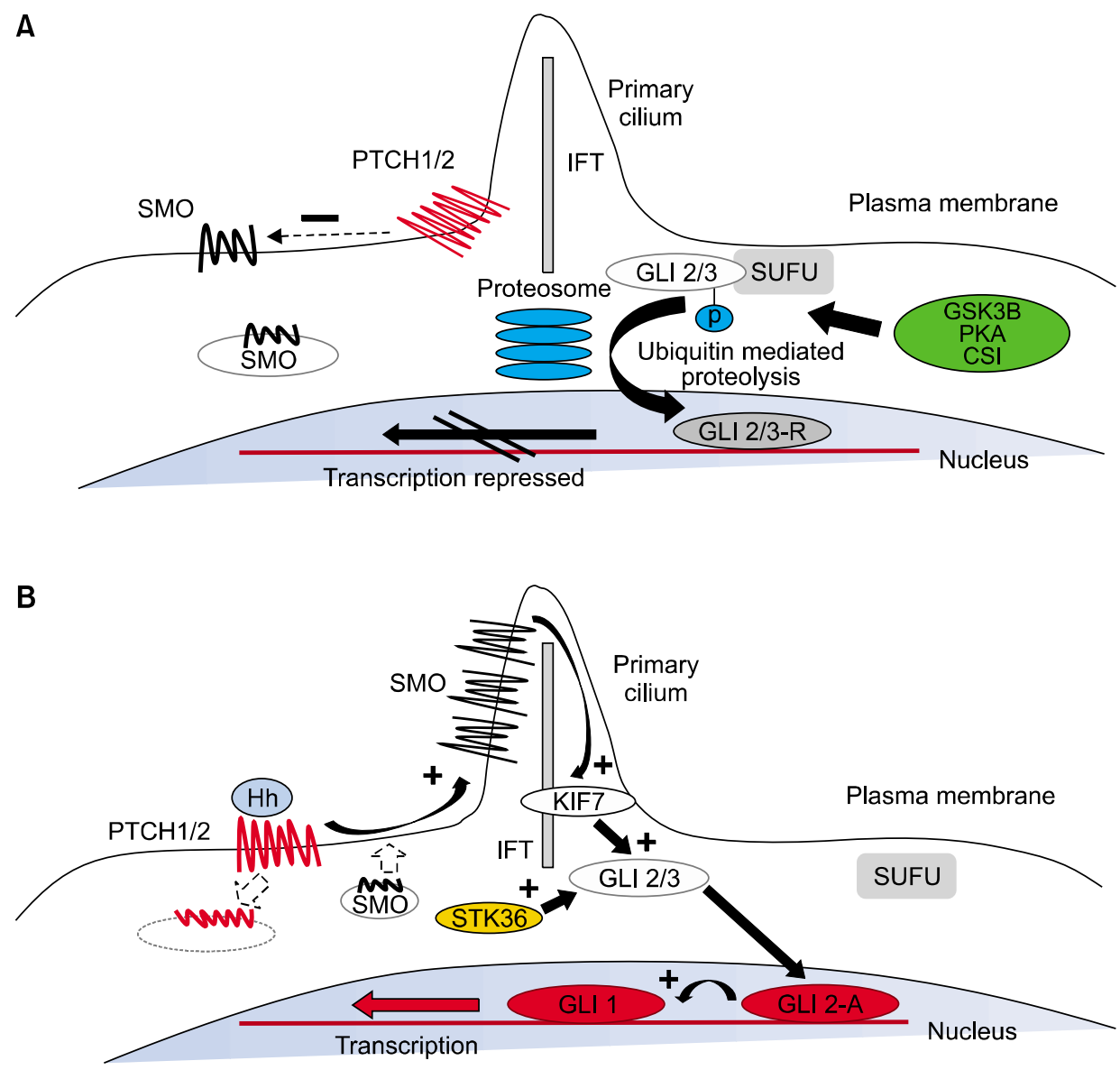

Figure 3. The mechanism of Hedgehog (Hh) signal transduction. (A) In the resting state, Pached (PTCH) $1 / 2$ is expressed on the plasma membrane and acts to repress Smoothened (SMO) activity by preventing its expression and localization to the primary cilium. GLI2/3 transcription factors are within a complex, including SUFU, an inhibitor of Hh signaling. This complex promotes nonspecific phosphorylation of the C-terminus by GSK3 $\beta$, CSI, and PKA, resulting in E3 ubiquitin ligase activity and subsequent partial proteosomal proteolysis to the C-terminal truncated repressor form. After translocation to the nucleus, the repressive form of GLI2 (GLI2-R) and GLI-3R potently inhibit the Hh transcriptional program. (B) Interaction of Hh ligand with PTCH promotes PTCH internalization and degradation and blocks the repression of SMO, causing its accumulation within the primary cilium. Active SMO in the primary cilium stabilizes the full-length forms of GLI2 (GLI2-A) and GLI3 (GLI3-A) and accentuates the effect of other positive regulators of $\mathrm{Hh}$ signaling, including serine threonine kinase 36 (STK36) and kinesin family member 7 (KIF7), which may be involved in translocation of GLI into the primary cilium. After translocation to the nucleus, GLI2-A potently activates transcription of downstream Hh targets, including GLI1 and PTCH1, and influences chromatin conformation, apoptosis, cell cycle activity, and differentiation (https://www.ncbi.nlm.nih.gov/pmc/articles/PMC3705708/figure/F1/). 
marrow transplant model. As a potential mechanism that leads to the upregulation of these $\mathrm{Hh}$ target genes in $\mathrm{BCR}-\mathrm{ABL}$ positive cells, SMO expression was significantly upregulated at both the mRNA and protein levels. SMO overexpression appears to be primarily BCR-ABL independent. Imatinib treatment of BCR-ABL positive bone marrow cultures only led to a minor decrease in Smoand Gli1 transcript levels. Therefore, it will be interesting to determine the precise mechanism that leads to SMO upregulation in BCR-ABL positive leukemic cells and whether this involves the interaction of leukemic cells with their microenvironment. Smo deficiency similarly decreased onset and latency of BCR-ABL-induced leukemia, and reduced frequency of leukemic stem cells ( $\operatorname{Lin}^{-}, \mathrm{Sca}-1^{+}, \mathrm{Kit}^{+}$). Together, both these studies carry important implications for the application of Hh inhibition therapy in targeting the CML stem cell compartment. The critical molecular effectors downstream of activated Hh signaling in CML, however, will need further investigation. The contribution of additional $\mathrm{Hh} / \mathrm{Gli}$ targets, such as the proto-oncogenes, BCL-2and CYCLIND, towards the growth of CML stem cells will need to be investigated.

\section{BIOLOGICAL COMPLEXITY OF CHRONIC MYELOID LEUKEMIA}

The molecular mechanisms underlying cancer progression are still uncertain, but most likely involve activation of oncogenic factors and/or inactivation of tumor suppressors. ${ }^{39}$ A plausible assumption is that BP is a multistep and time-dependent process initiated by both BCR-ABL1-dependent (Fig. 4) and-independent DNA damage associated with inefficient and unfaithful DNA repair in CML-CP. CML-CP, if facilitated by an increased level of BCR-ABL1 activity, leads to selection of one or more CML-BP

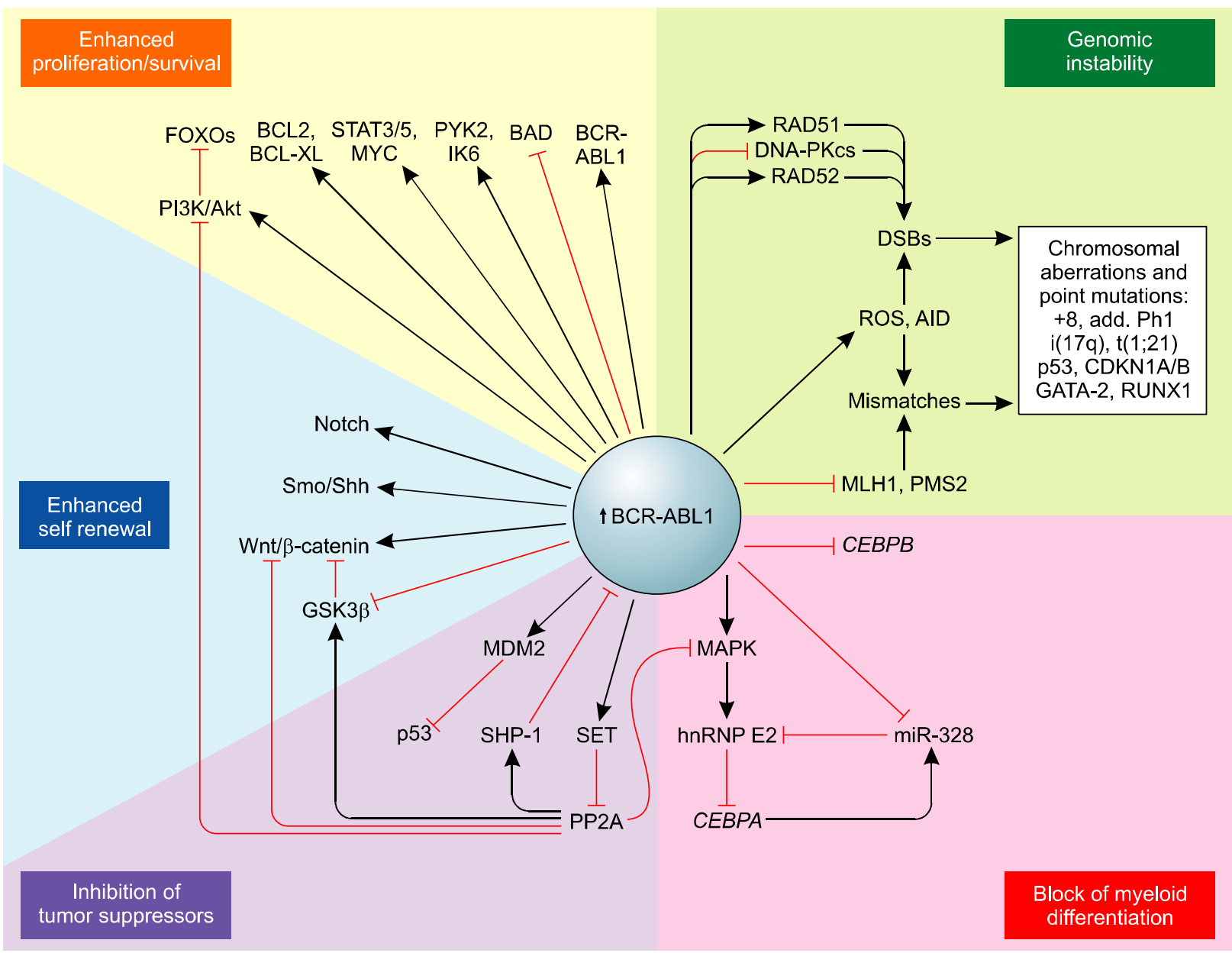

Figure 4. The potential BCR-ABL1-dependent molecular mechanisms leading to chronic myeloid leukemia disease progression (https://www. ncbi.nlm.nih.gov/pmc/articles/PMC2898591/). BAD, BCL2 antagonist of cell death; DNA-PKcs, DNA-dependent protein kinase, catalytic subunit; FOXO, fork head box O; IK6, Ikaros 6; miR-328, microRNA-328; MLH1, mutL homolog 1; PMS2, post meiotic segregation increased 2; RAD51, RecA homolog in Escherichia coli; RAD52, RAD52 homolog (Saccharomyces cerevisiae); Shh, Sonic Hh; wnt//-catenin, wingless-int1/beta-catenin. 
clones.

The relatively high BCR-ABL1 expression/activity in CML-CP $\mathrm{CD} 34^{+} \mathrm{CD} 38^{-}$stem cells and/or $\mathrm{CD} 34^{+}$early progenitors compared with more committed progenitors, which is further markedly increased in CML-BP $\mathrm{CD} 34^{+}$progenitors, results in the following: enhancement of proliferation/survival pathways, increased genomic instability, activation of pathways blocking in myeloid differentiation, acquisition of the ability for self-renewing, and inhibition of tumor suppressors with broad cell regulatory functions.

The genetic lesions observed in CML-BP patients in the past and now since the introduction of TKIs mostly include the presence of additional chromosomes, gene deletions, gene insertions, and/or point mutations (including BCR-ABL1 mutations), ${ }^{40,41}$ but patterns differ in myeloblastic and lymphoblastic transformations.

\section{CONCLUSIONS}

There has been an increased understanding of several key genetic changes that drive CML. In this review, we described and critically evaluated different technologies used to detect $B C R$-ABL1 transcripts in patients with CML. We focused on the comparison between current and new technologies. We discussed potential advantages of these new technologies for monitoring response to CML management. We suggest that molecular and NGS analyses may transform our approach to molecular monitoring of cancers in the next 5 to 10 years, not only for CML but also for other leukemias and solid cancers. In addition to analytical validation, the clinical relevance of better ability to detect and accurately quantify low levels of residual transcripts from cancer cells needs to be evaluated in clinical trials. The next challenge for investigators will be to bridge the gap between discoveries made in the laboratory, and identify novel therapies that target these molecular alterations to improve outcomes.

\section{CONFLICTS OF INTEREST}

No potential conflicts of interest were disclosed.

\section{REFERENCES}

1. Apperley JF. Part II: management of resistance to imatinib in chronic myeloid leukaemia. Lancet Oncol 2007;8:1116-28.

2. O'Brien SG, Guilhot F, Larson RA, Gathmann I, Baccarani M, Cervantes $\mathrm{F}$, et al. Imatinib compared with interferon and low-dose cytarabine for newly diagnosed chronic-phase chronic myeloid leukemia. N Engl J Med 2003;348:994-1004.

3. Rowley JD. Letter: a new consistent chromosomal abnormality in chronic myelogenous leukaemia identified by quinacrine fluorescence and Giemsa staining. Nature 1973;243:290-3.

4. Druker BJ, Tamura S, Buchdunger E, Ohno S, Segal GM, Fanning $S$, et al. Effects of a selective inhibitor of the Abl tyrosine kinase on the growth of Bcr-Abl positive cells. Nat Med 1996;2:561-6.

5. Traxler P, Bold G, Buchdunger E, Caravatti G, Furet P, Manley P, et al. Tyrosine kinase inhibitors: from rational design to clinical trials. Med Res Rev 2001:21:499-512.

6. Hughes TP, Hochhaus A, Branford S, Müller MC, Kaeda JS, Foroni $\mathrm{L}$, et al. Long-term prognostic significance of early molecular response to imatinib in newly diagnosed chronic myeloid leukemia: an analysis from the International Randomized Study of Interferon and STI571 (IRIS). Blood 2010;116:3758-65.

7. Weerkamp F, Dekking E, Ng YY, van der Velden VH, Wai H, Böttcher S, et al. Flow cytometric immunobead assay for the detection of BCR-ABL fusion proteins in leukemia patients. Leukemia 2009;23:1106-17.

8. Seabright M. A rapid banding technique for human chromosomes. Lancet 1971;2:971-2.

9. Machova Polakova K, Koblihova J, Stopka T. Role of epigenetics in chronic myeloid leukemia. Curr Hematol Malig Rep 2013;8:28-36.

10. Katoh M. Functional and cancer genomics of ASXL family members. Br J Cancer 2013;109:299-306.

11. Carbuccia N, Murati A, Trouplin V, Brecqueville M, Adélaïde J, Rey $\mathrm{J}$, et al. Mutations of ASXL1 gene in myeloproliferative neoplasms. Leukemia 2009:23:2183-6.

12. Gelsi-Boyer V, Trouplin V, Adélaïde J, Bonansea J, Cervera N, Carbuccia N, et al. Mutations of polycomb-associated gene ASXL1 in myelodysplastic syndromes and chronic myelomonocytic leukaemia. Br J Haematol 2009;145:788-800.

13. Soroush A, Farshchian N, Komasi S, Izadi N, Amirifard N, Shahmohammadi A. The role of oral contraceptive pills on increased risk of breast cancer in iranian populations: a meta-analysis. J Cancer Prev 2016:21:294-301.

14. Cloos PA, Christensen J, Agger K, Helin K. Erasing the methyl mark: histone demethylases at the center of cellular differentiation and disease. Genes Dev 2008;22:1115-40.

15. Abdel-Wahab O, Pardanani A, Patel J, Wadleigh M, Lasho T, Heguy A, et al. Concomitant analysis of EZH2 and ASXL1 mutations in myelofibrosis, chronic myelomonocytic leukemia and blast-phase myeloproliferative neoplasms. Leukemia 2011;25:1200-2.

16. Schmidt M, Rinke J, Schäfer V, Schnittger S, Kohlmann A, Obstfelder E, et al. Molecular-defined clonal evolution in patients with chronic myeloid leukemia independent of the BCR-ABL status. Leukemia 2014:28:2292-9.

17. Kerenyi MA, Shao Z, Hsu YJ, Guo G, Luc S, O'Brien K, et al. Histone demethylase Lsd1 represses hematopoietic stem and progenitor cell signatures during blood cell maturation. Elife 2013;2:e00633.

18. Li F, Mao G, Tong D, Huang J, Gu L, Yang W, et al. The histone mark H3K36me3 regulates human DNA mismatch repair through its interaction with MutS $\alpha$. Cell 2013;153:590-600.

19. Wimmer K, Etzler J. Constitutional mismatch repair-deficiency syndrome: have we so far seen only the tip of an iceberg? Hum Genet 2008; 124:105-22.

20. Oktem G, Sercan O, Guven U, Uslu R, Uysal A, Goksel G, et al. Cancer stem cell differentiation: TGF $\beta 1$ and versican may trigger 
molecules for the organization of tumor spheroids. Oncol Rep 2014:32:641-9.

21. Akard LP, Wang YL. Translating trial-based molecular monitoring into clinical practice: importance of international standards and practical considerations for community practitioners. Clin Lymphoma Myeloma Leuk 2011;11:385-95.

22. Ernst T, Chase A, Zoi K, Waghorn K, Hidalgo-Curtis C, Score J, et al. Transcription factor mutations in myelodysplastic/myeloproliferative neoplasms. Haematologica 2010;95:1473-80.

23. Itzykson R, Kosmider O, Renneville A, Gelsi-Boyer V, Meggendorfer $\mathrm{M}$, Morabito $\mathrm{M}$, et al. Prognostic score including gene mutations in chronic myelomonocytic leukemia. J Clin Oncol 2013;31:2428-36.

24. Movafagh A, Hajifathali A, Zamani M. Secondary chromosomal abnormalities of de novo acute myeloid leukemia: a first report from the Middle East. Asian Pac J Cancer Prev 2011;12:2991-4.

25. Grossmann V, Kohlmann A, Eder C, Haferlach C, Kern W, Cross $\mathrm{NC}$, et al. Molecular profiling of chronic myelomonocytic leukemia reveals diverse mutations in $>80 \%$ of patients with TET2 and EZH2 being of high prognostic relevance. Leukemia 2011:25:877-9.

26. Movafagh A, Hajifathali A, Isfahani F, Attarian H, Ghadiani M, Rezvani H, et al. Geographic heterogeneity of cytogenetic characteristics of acute myeloid leukemia in the early detection: a comparative study of iranian and indian adult patients. IJCP 2009;2:85-9.

27. Kohlmann A, Grossmann V, Klein HU, Schindela S, Weiss T, Kazak B, et al. Next-generation sequencing technology reveals a characteristic pattern of molecular mutations in $72.8 \%$ of chronic myelomonocytic leukemia by detecting frequent alterations in TET2, CBL, RAS, and RUNX1. J Clin Oncol 2010;28:3858-65.

28. Varma N, Varma S, Movafagh A, Garewal G. Unusual clonal cytogenetic abnormalities in aplastic anemia. Am J Hematol 1995:49:256-7.

29. Daver N, Strati P, Jabbour E, Kadia T, Luthra R, Wang S, et al. FLT3 mutations in myelodysplastic syndrome and chronic myelomonocytic leukemia. Am J Hematol 2013;88:56-9.

30. Laborde RR, Patnaik MM, Lasho TL, Finke CM, Hanson CA, Knudson RA, et al. SETBP1 mutations in 415 patients with primary myelofibrosis or chronic myelomonocytic leukemia: independent prognostic impact in CMML. Leukemia 2013;27:2100-2.

31. Damm F, Itzykson R, Kosmider O, Droin N, Renneville A, Chesnais V, et al. SETBP1 mutations in 658 patients with myelo- dysplastic syndromes, chronic myelomonocytic leukemia and secondary acute myeloid leukemias. Leukemia 2013;27:1401-3.

32. Itzykson R, Solary E. An evolutionary perspective on chronic myelomonocytic leukemia. Leukemia 2013;27:1441-50.

33. Chu S, Xu H, Shah NP, Snyder DS, Forman SJ, Sawyers CL, et al. Detection of BCR-ABL kinase mutations in $\mathrm{CD} 34+$ cells from chronic myelogenous leukemia patients in complete cytogenetic remission on imatinib mesylate treatment. Blood 2005;105:2093-8.

34. Lee KE. Immunohistochemical Assessment of O(6)-Methylguanine-DNA Methyltransferase (MGMT) and Its Relationship with p53 Expression in Endometrial Cancers. J Cancer Prev 2013; 18:351-4.

35. Shah NP, Nicoll JM, Nagar B, Gorre ME, Paquette RL, Kuriyan J, et al. Multiple BCR-ABL kinase domain mutations confer polyclonal resistance to the tyrosine kinase inhibitor imatinib (STI571) in chronic phase and blast crisis chronic myeloid leukemia. Cancer Cell 2002;2:117-25.

36. Breccia M, Diverio D, Pane F, Nanni M, Russo E, Biondo F, et al. Discontinuation of imatinib therapy after achievement of complete molecular response in a $\mathrm{Ph}(+) \mathrm{CML}$ patient treated while in long lasting complete cytogenetic remission (CCR) induced by interferon. Leuk Res 2006;30:1577-9.

37. Rousselot P, Huguet F, Rea D, Legros L, Cayuela JM, Maarek O, et al. Imatinib mesylate discontinuation in patients with chronic myelogenous leukemia in complete molecular remission for more than 2 years. Blood 2007; 109:58-60.

38. Calabretta B, Perrotti D. The biology of CML blast crisis. Blood 2004:103:4010-22

39. Yamamoto M, Kakihana $\mathrm{K}$, Kurosu $\mathrm{T}$, Murakami N, Miura O. Clonal evolution with inv(11)(p15q22) and NUP98/DDX10 fusion gene in imatinib-resistant chronic myelogenous leukemia. Cancer Genet Cytogenet 2005;157:104-8.

40. Fabarius A, Haferlach C, Müller MC, Erben P, Lahaye T, Giehl M, et al. Dynamics of cytogenetic aberrations in Philadelphia chromosome positive and negative hematopoiesis during dasatinib therapy of chronic myeloid leukemia patients after imatinib failure. Haematologica 2007;92:834-7.

41. Roche-Lestienne C, Deluche L, Corm S, Tigaud I, Joha S, Philippe $\mathrm{N}$, et al. RUNX1 DNA-binding mutations and RUNX1-PRDM16 cryptic fusions in BCR-ABL+ leukemias are frequently associated with secondary trisomy 21 and may contribute to clonal evolution and imatinib resistance. Blood 2008;111:3735-41. 